\title{
Unity of opposites? Chronic fatigue syndrome and the challenge of divergent perspectives in guideline development
}

\author{
Charlotte Smith, Simon Wessely
}

Department of Psychological Medicine, Institute of Psychiatry, Weston Education Centre, London, UK

\section{Correspondence to} Dr Charlotte Smith, Department of Psychological Medicine, Institute of Psychiatry, Weston Education Centre, Cutcombe Road, London SE5 9RJ, UK; charlotte.smith@slam.nhs.uk

Received 11 May 2012 Revised 19 September 2012 Accepted 17 October 2012 Published Online First 17 November 2012

\section{SLinked}

- http://dx.doi.org/10.1136/ jnnp-2012-303857

- http://dx.doi.org/10.1136/ jnnp-2012-304208

- http://dx.doi.org/10.1136/ jnnp-2012-304824

To cite: Smith C, Wessely S I Neurol Neurosurg Psychiatry 2014;85: 214-219.

\section{ABSTRACT}

Guideline development by its nature is a process and method of integration and synthesis of information, be it originating from research, evidence-based medicine, clinical findings, patient experience and/or individual narratives of an illness or disease. In the majority of cases, it can be assumed that this information and these ideas are travelling in the same direction; however, it is possible that the objective and subjective cannot be synthesised, and appear mutually contradictory. In this commentary, an example of where this might be the case has been analysed: a report published by the Scottish Public Health Network, a Health Care Needs Assessment of Services for people living with myalgic encephalomyelitis (ME)/chronic fatigue syndrome (CFS). It appears from reflection and analysis of this document that this process may indeed have gone awry. We propose that, if followed, this document would lead to the adoption of dangerous diagnostic criteria for $\mathrm{ME} / \mathrm{CFS}$, as well as preventing patients from making informed decisions about treatment options, and discouraging clinicians from following evidence-based medicine and recommending proven treatments for ME/CFS, because of potential implications for future commissioning. This commentary seeks to highlight some of the problems, contradictions and unintended consequences of a divergence between patient perspectives and evidence-based medicine despite probably sharing the same aim, that of improving patient care and striving for better understanding and better treatments for disease.

Clinicians are presented with a wealth of research, often looking at the statistical, the significant, the evidence base, the objective measures, objective outcomes and quantitative data. They are then faced with patients, the people to whom this evidence may apply, holding in mind the individual narrative, the subjective, the qualitative and the patient experience. Arguments about the relative merit of the one versus the other have largely gone the way of nature versus nurture polemics in genetics-few doubt that both are needed. This is, however, based on an assumption that both the subjective and objective are travelling in the same direction and share the same aims. It is assumed that research findings should, and will in some way, complement the narrative view and lead to a collaborative approach to new ideas, new understandings and finally better treatments for illnesses and disease.
But what happens if this method and process of integration goes awry? What happens when the objective and the subjective not only cannot be synthesised, but appear mutually contradictory? What happens when, at first sight, the results of a considerable quantity of what we now call 'evidence-based medicine' firmly point in one direction, while the narrative and individual accounts, at least those that are readily available, perhaps even more vehemently say the opposite. How and why can this happen, and does it matter?

Guidelines and the process by which they are formulated are an important attempt to formalise the convergence of the narrative and the scientific. If the process is carried out successfully, the result should be a balanced, complementary, mixed method review of what is known of a condition, based on the scientific evidence while taking into account individuality. ${ }^{1}$

In this essay, we wish to draw attention to a particular example of this phenomenon, and will argue that it does matter, not least to those for whom the entire process of evidence synthesis is finally designed to help, patients themselves.

The example that we have chosen is a report produced in September 2010 by the Scottish Public Health Network (ScotPHN) entitled 'Health care needs assessment of services for people living with myalgic encephalomyelitis (ME)-chronic fatigue syndrome (CFS), ${ }^{2}$ with a short version for patients produced in September 2011. ${ }^{3}$ This formal review of healthcare needs was commissioned by the Scottish Government to undertake a review of the scientific literature, including different national guidelines for diagnosis and treatment, and to establish the needs of individuals with ME/CFS. The overarching objective was to understand service user needs and to undertake an assessment of subsequent care and service provision. This has potentially important implications for subsequent commissioning and funding of services by NHS Scotland. The ScotPHN's aim was to complete a national project of needs assessment that would incorporate the views of clinicians and patients from across Scotland.

$\mathrm{ME} / \mathrm{CFS}$ is historically a condition that has attracted much debate and overt conflict. The authors of the report themselves accept that the area is one that continues to attract more than its fair share of controversy. There are many different views-from patients, professionals and carers, and the patient groups and activist organisations which 
have been set up to represent patient views, particularly in the areas of approach to diagnosis, treatment and future research. ${ }^{i}$

Unfortunately, it is our view that this report does not help resolve the controversy and may further confuse both patients and clinicians. We also argue that this has implications beyond the troubled field of ME/CFS, since it highlights a possible unintended but still regrettable consequence of the collision of the two worlds that we outlined in the opening paragraph. Overall, we argue that the results are unsustainable conclusions, with implications that are at best misleading and at worst harmful for patients and clinicians.

The first example of this is in the area of diagnosis. The ScotPHN report has recommended that Scotland uses two separate categories for diagnosis, one diagnosis of ME/CFS, based on the National Institute for Health and Clinical Excellence (NICE) 2007 criteria, and a separate diagnosis of ME, based on the Canadian Consensus Document Criteria. ${ }^{2}$ They suggest that this is 'a pragmatic approach to allow clinicians to adopt an approach to diagnosis that can ensure that those individuals for whom CFS exists are identified as rapidly as possible and also allow for more focussed assessment and review to confirm a diagnosis of ME'. ${ }^{2}$ They continue to simply state 'Diagnostic issues are more fully explored in the Scottish Good Practice Statement (SGPS) on ME-CFS'. ${ }^{2}$ The report outlines with respect to the Canadian guidelines that it 'emphasises the neurological features of the condition and the post-exertion fatigue/malaise which more psychiatrically-based definitions under emphasise'. ${ }^{2}$

The Canadian consensus document was originally developed in response to pressure from the National ME/FM Action Network of Canada. This patient group had disseminated a questionnaire to doctors in Canada and found that the responding doctors felt clearer clinical definitions as well as diagnostic and treatment protocols would be useful to help manage their patients with ME/CFS. The patient group approached the then Minister of Health who then approached Health Canada to oversee a consensus document to address this. The report states that Health Canada selected an Expert Consensus Panel for $\mathrm{ME} / \mathrm{CFS}$, and some criteria regarding nominations and experience were recommended; however, although the panel members fulfilled these criteria, they consisted of clinicians specifically selected by the patient group. ${ }^{4}$ Importantly, it was made clear that the members of the panel had complete autonomy over their consensus document. There are many general problems with the Canadian consensus document that we could discuss, arguably the most important of which is that the guidelines were developed without reference to any standard methodology that would usually be utilised for guideline development. The document was published in a journal that is no longer in existence, and the group who developed the guideline declare that they were in part funded by Biovail Pharmaceuticals, subsequently merged with Valeant Pharmaceuticals International, a firm that specialises in neurological and central nervous system drug development and production. ${ }^{5}$ The criteria that the Canadian consensus group have produced have not been operationally defined or validated. ${ }^{4}$ Many of the recommendations and assertions that are made in the document are not supported by published or peer-reviewed evidence, and the report holds an open stance of a neurological view of $\mathrm{ME} / \mathrm{CFS}$, also not

${ }^{\mathrm{i}}$ Nomenclature is confusing in this area. Chronic fatigue syndrome (CFS) is the term usually used by doctors and in the professional literature, whereas myalgic encephalomyelitis (ME) is preferred in the UK (but not US) media and by some patient groups. referenced or supported by the evidence. The document itself seems to have fallen foul of the key to guideline development in that there is a lack of robust peer review. The process of developing this document is in itself flawed, a result of patient group pressure on politicians who then outsource the work to a panel the patient group select, leading to a one-sided document.

Besides the questionable practicality of using two separate definitions for CFS and ME, it is true that there is a major discrepancy in the neurological criteria that the Canadian criteria and NICE have recommended, with the NICE guidelines referring to 'cognitive dysfunction, such as difficulty thinking, inability to concentrate, impairment of short term memory, and difficulties word finding, planning/organising thoughts and information processing ${ }^{6}$ they also recognise 'dizziness and/or nausea' ${ }^{6}$ as possible symptoms. The Canadian guidelines include in their section on 'Neurological/cognitive manifestations...confusion, disorientation, inability to focus vision...photophobia' ${ }^{4}$ and also state that 'Ataxia, muscle weakness and fasciculations are common'.

The ScotPHN report makes a note following their recommendation to use the Canadian consensus criteria that 'The HCNA acknowledges that the SGPS is the recognised clinical guidance on the diagnostic approach to ME-CFS. This is important as the SGPS also addresses the concerns raised by the Scottish Neurosciences Council regarding the use of the Canadian Criteria'. ${ }^{2}$ The Scottish Neurosciences Council published a forthright response to the SGPS draft advising specific concerns regarding adopting the Canadian consensus document. They state 'The Scottish Neuroscience Council takes the view that the 'hard' neurological signs of ataxia or fasciculations never occur in ME. Where these signs do occur, they have very specific clinical implications'. 7 The Council warn that 'There is a strong concern that by including these symptoms and signs in its core description of the condition, the statement would lead to misdiagnosis, both of those with ME-CFS and with other unrelated serious neurological diseases'. ${ }^{7}$ Owing to this, the Scottish Good Medical Practice working group revised their report, which now states that 'neurological examination should be carried out to exclude specific neurological abnormalities such as: obvious muscle wasting, ptosis, upper motor neurone signs, ataxia, fasciculations, absent reflexes. If any of these abnormalities are present, neurological specialist referral is indicated. Muscle twitches and spasms occur and weakness is also common in ME-CFS because of pain and fatigue, but normal power is usually possible even if only for a few seconds'. ${ }^{8}$ They also describe objective neurological signs as a 'red flag', having alternative diagnostic implications. The SGPS have not in fact endorsed the use of any particular criteria for diagnosing ME/ CFS, and in reference to the Canadian criteria they only state that 'When the Canadian Consensus Document definition is used to assist the diagnosis and management of ME-CFS, clinicians should carefully adhere to this specific neurological referral recommendation'. ${ }^{8}$ It seems as though the SGPS, which is, as the ScotPHN Health Care Needs Assessment Group state themselves is the guiding document for current practice, have remained ambiguous regarding which criteria should be adopted in Scotland, for reasons that remain opaque. If the purpose was to please patient groups who strongly supported the view that ME/CFS was neurological, this has been negated with the caveat that neurological signs are not indeed recognised as part of CFS or ME. This is the view of most neurologists: in a 2011 survey of neurologists working in the UK, 84\% did not consider that ME or CFS was a neurological condition. ${ }^{9}$

If one accepts, as most neurologists do, that some of the signs and symptoms that are held by the Canadian consensus criteria 
to be incompatible with a diagnosis of ME/CFS, then the adoption of those same criteria by the ScotPHN Health Care Needs Assessment Group encourages poor practice and would, if implemented, have a detrimental impact on patient care. It is clear that there is strong support from some quarters for the adoption of the Canadian criteria-a vote for example of members of the ME Association supported this, against the advice of their own medical advisor. Indeed, the Canadian criteria have become a litmus test among some sections of the patient community-if you are for it, you are supporting a neurological or neuroimmune view of the illness, and if you are against it, you must be in favour of a psychological/psychiatric view. But this is not a matter of opinion, preference or politics. Either fasciculations are compatible with a diagnosis of ME/CFS or they are not. Attempting to synthesise patient views into the discourse regarding which criteria should be used to identify patients clinically has led to dangerous criteria being adopted, which increases the risk of misdiagnosis with all that might imply. Even if this is supported by the majority of those who make their voices heard on this issue, it still may not be in the interests of the majority or indeed entirety of all patients. We took a straw poll of the members of two lists of multiprofessional NHS clinicians who regularly see ME/CFS patients and probably represent the majority of NHS professionals active in the field, and only a handful could recall any patients who had raised the issue of the Canadian guidelines or showed any interest in the subject. It seems not to be an issue for the majority of $\mathrm{ME} / \mathrm{CFS}$ sufferers receiving treatment within the NHS. ${ }^{\text {ii }}$

The second example concerns treatment. Exercise is an effective therapy for many chronic diseases. ${ }^{10}$ A 2006 review of the literature relating to exercise and many chronic diseases including ME/CFS showed that there was moderate evidence for exercise improving symptoms specific to ME/CFS and improving physical fitness and strength; furthermore, no contraindications to exercise in $\mathrm{ME} / \mathrm{CFS}$ were identified. ${ }^{10} \mathrm{~A}$ peer-reviewed US systematic review in 2001 into strategies and interventions for treatment and management of ME/CFS concluded that the 'interventions which have shown promising results include cognitive behavioural therapy (CBT) and graded exercise therapy $(\mathrm{GET})^{11}$; these were in fact the only two interventions shown to be both effective and safe. Following this, a 2004 Cochrane review of exercise therapy in ME/CFS concluded that there was 'encouraging evidence that some patients may benefit from exercise therapy and no evidence that exercise therapy may worsen outcomes'. ${ }^{12}$ In 2006, a UK systematic review of the treatments, management and rehabilitation of patients also found that 'GET and CBT appeared to reduce symptoms and improve function based on evidence from RCTs. For most other interventions, evidence of effectiveness was inconclusive and some interventions were associated with significant adverse effects' ${ }^{13}$; again

\footnotetext{
iiThis is based on responses to emails sent to two lists of NHS professionals routinely involved in treating the condition. While we acknowledge that this is only a straw poll, it does highlight the different experiences reported by NHS staff who treat ME/CFS compared with those that emerge from activist groups. One of the respondents to the poll runs one of the largest adult services in the UK, with over 500 new referrals a year and over 10 years of experience working within the service; their experience was that they had 'never had this as an issue'. A typical response out of many similar was: 'I have come across this only once or twice, and this has usually been due to patients contacting doctors outside the UK or where the diagnosis has been inappropriately given by other services. Most patients are more concerned to know what I think is wrong with them and what I am going to do to help them'.
}

CBT and GET were the only treatments for which there was evidence of significant efficacy. This was supported by two subsequent systematic UK reviews completed and updated by the Centre for Reviews and Dissemination ${ }^{14} 15$ and by a Lancet review, the authors of which concluded that 'CBT and GET are the only interventions found to be beneficial'. ${ }^{16}$ In this review, the authors also present the evidence for rejecting multiple other treatment modalities tested, including in randomised controlled trials, ranging from pharmacological, supplementary, complementary and other interventions. ${ }^{16}$ This evidence for the beneficial effect of CBT and GET has been replicated in a meta-analysis extending the review to evidence of overall and outcome-specific effects, comparing the treatments and addressing the methodological limitations of other reviews. ${ }^{17}$

The ScotPHN report states that, in conclusion to their literature search regarding GET, 'Views on graded exercise programmes varied. Although it was acknowledged that a degree of exercise relating directly to each individual's level of energy and/or pain may be beneficial, the international summary included a cautionary note that graded exercise programmes had recently been identified as harmful'. ${ }^{2}$ While they recognise that GET is recommended by the NICE guidelines $2007,{ }^{6}$ much weighting of the discussion regarding GETs safety and efficacy is taken from the Action for ME Scoping Study-for example, they state that 'a significant proportion of people with ME-CFS who participated in the Action for ME Scoping Study said that GET made them worse with roughly $75 \%$ of those who reported undertaking GET describing themselves as getting worse'. ${ }^{2}$ However, it is noted that most of these responders did not actually participate in exercise that was graded or guided by an appropriate therapist, and there are limitations as to how these views can be generalised to the general population of patients with $\mathrm{ME} / \mathrm{CFS}$, since no diagnosis was established. There is also no evaluation of the wealth of other contradictory well-conducted research, including randomised controlled trials and systematic reviews of treatment in existence that provide evidence for the efficacious and safe use of GET in ME/CFS. The comments regarding the lack of safety are not evidence based-in fact, the evidence from controlled studies shows the opposite. ${ }^{18}$ There is no particular reason why graded exercise carried out under appropriate professional supervision should be harmful, and indeed, as stated previously, it is used in the rehabilitation of numerous cardiac, neurological and many other disorders to successful effect. ${ }^{10}$ One would need compelling evidence to overturn these observations, and none are provided.

With regard to CBT, the authors of the ScotPHN report refer to 'highly differing views on the role of CBT...Within Scotland the CMO's Short life working group commented that whilst there was a role for CBT in the management of ME-CFS for some patients, it is not a technique that should be recommended to every patient'. 2 This plays down the role that CBT has in the treatment of ME/CFS, and gives the impression that there is no evidence either way as to effectiveness or safety in ME/CFS when in fact its role has been demonstrated in various standardised systematic reviews of the literature. Again the discussion appears to refer extensively to the survey of patient groups, which are inevitably biased against those who have improved, contain heterogeneous groups of people probably with a wide variety of diagnoses, and a bias as in all self-help groups towards those with poor prognosis. As with the GET literature, there is little evidence regarding which interventions patients undertook. Given the general lack of provision of both CBT and GET for most disorders, and ME/CFS in particular, across the UK, it 
is implausible that the majority who responded actually received either intervention as it is defined in the studies and guidelines.

Furthermore, the literature review that has been undertaken appears to give a similarly unbalanced appraisal, and seems to have compared national guidelines, without conducting its own comprehensive literature review, as would be standard for guideline production. It is unclear if any independent literature review has been carried out; similarly, there is no reference to procedures the group took when reviewing the literature, and therefore it cannot be replicated. The critical appraisal of the literature that has been presented is limited, and there is poor clarity regarding the different grades of evidence used; thus the reader may be left with the impression that all the evidence presented is of equal validity.

One reason why the ScotPHN guideline shies away from coming to the same conclusions as all previous efforts at synthesis directly relates to the controversy and politics of ME/CFS. The report specifically discusses the NICE guidelines published in 2007, which recommend the use of CBT and GET for mild to moderate $\mathrm{ME} / \mathrm{CFS},{ }^{6}$ this based on a literature review that was carried out as part of the gold standard guideline development process. The ScotPHN report, however, in conclusion to the review of evidence of treatment and management that it is hard to see how... a formal Scottish Inter-Collegiate Guideline Network (SIGN) guideline be developed. It is probable that such an approach...would not be able to draw on a sufficient body of research to complete...robust, quality assured, systematic analysis'. ${ }^{2}$ It is hard to see the logic of this and why ScotPHN felt that there was insufficient reason for a SIGN review, given that many distinguished bodies and organisations, including NICE, have done just that. In our opinion, this might be due to their own admission that the results and conclusions may not be acceptable to certain stakeholders. They suggest, 'where such an approach was attempted by NICE, it (was) subject to challenges from stakeholders and interested parties on the grounds of the research that was considered for inclusion'. However, they fail to set the context of that challenge, which resulted in a judicial review. In a robust judgement, claims that the NICE guidelines were irrational and the panel biased were emphatically rejected. ${ }^{19} \mathrm{Mr}$ Justice Simon stated that the allegations were without foundation, that the evidence had been considered appropriately, that there had been no attempt to ignore the risks of CBT and GET, and that the panel had acted fairly. ${ }^{19}$ Furthermore, he concluded that, even if he had been persuaded that the recommendation was flawed on those grounds, he 'would not have made an order quashing the recommendation as this would have resulted in no recommended treatment for those suffering from CFS/ME'. ${ }^{19}$

Since the publication of the main guidelines, the results of what is the most important trial of CBT and GET have become available. The ScotPHN main guideline could only acknowledge that the trial was ongoing, but the full results were published in March 2011, some 6 months before the date of the patient guidelines produced by the ScotPHN. ${ }^{3}$ They form no conclusions about the treatment options in the main Needs Assessment Document, ${ }^{2}$ and the ScotPHN short report for patients makes no reference at all to the only two validated treatments for ME/ CFS, namely CBT and GET. ${ }^{3}$ The PACE trial is a very large $(n=640)$ and well conducted (1 year follow-up rate of 95\%) multicentre randomised study, funded by the Medical Research Council, Department of Health and Department of Work and Pensions, and ironically also the Scottish Chief Scientist's office, and one of whose major centres included Edinburgh. It tested the effectiveness and safety of adaptive pacing therapy, CBT,
GET and specialist medical care. ${ }^{18}$ One of its stated aims was to test the safety of the different treatments, in direct response to claims that either CBT or GET were dangerous. ${ }^{20}$ The results confirmed and added to the existing body of research, showing that 'CBT and GET can safely be added to specialist medical care to moderately improve outcomes for CFS' ${ }^{18}$ The issue of safety is now resolved beyond reasonable doubt-all four management approaches had an excellent safety profile, with little differences between any. ${ }^{18}$ The trial concluded that 'this finding is important and should be communicated to patients to dispel unnecessary concerns about the possible detrimental effects of CBT and GET'. ${ }^{21}$ This largest ever trial in ME/CFS and its treatment alone should be a reason for ScotPHN to reconsider their review and conclusions of this area.

CBT and GET continue to be the two recognised recommended therapies endorsed by mainstream patient advice resources, including the Mayo Clinic ${ }^{22}$ and BUPA. ${ }^{23}$ CBT and GET are also indeed endorsed in Scotland by their own Scottish good medical practice statement on $\mathrm{ME} / \mathrm{CFS},{ }^{8}$ which cite these as the only two therapies with level $1+$ evidence of interventions that are proven to be of benefit. ${ }^{8}$ We think there is a case that, despite what was clearly a great deal of effort, in the end the ScotPHN did not reflect a fair and balanced summary of the evidence when it was first drafted, nor after the results of PACE became available.

But does this matter? Yes. Without a dispassionate and clear account of the evidence-which must include an impartial summary of the possible risks and benefits of CBT and GET-the risk is that patients are being denied information about efficacy and availability of these treatments and therefore are not able to make an informed decision about whether the treatment is acceptable to them, impeding their right to exercise choice. No one has ever advocated that CBT or GET should be made compulsory, but surely patients must be able to make informed choices on the basis of an unbiased quality assessment of the evidence, after which they are free to choose either way. The conclusions drawn in this report would also deprive Scottish clinicians who wish to follow evidence-based medicine the ability to access these treatments for their patients by hindering their commissioning.

In fact, while the ScotPHN document may reflect and integrate views of a certain section of the ME community, these views may not be typical of others who belong to the established patient charities operating in this area. A patient survey that contradicts the views of the surveys quoted in the report was carried out by Action for ME. This report summarised that $84.9 \%$ of patients thought that Action for ME should campaign to save existing services, stating that 'all of the treatments listed benefit at least some people'. ${ }^{24}$ The majority of patients also wanted Action for ME to campaign for more services across the UK to improve access, and the majority felt that GET and CBT should be available on the NHS, along with other therapies including pacing, graded activity therapy, fatigue management and medication. ${ }^{24}$

Whatever one's views on the topic of ME/CFS, even the most passionate critic of psychiatry or psychological medicine would agree that this document is not a fair reflection of what is known. The ScotPHN appear to have marginalised psychiatric and psychological perspectives of ME/CFS. Their summaries, for example, of aetiology do not explore any of the established biopsychosocial risk factors for ME/CFS as demonstrated in a recent meta-analysis from the Centre for Reviews and Dissemination. ${ }^{16}$ Many of those who are not part of the controversy may find this baffling.

What lies behind this? We cannot know this conclusively, or find out from any publicly available document. It strikes us, 
however, that internet searches of patient group websites and forums reveal a stream of antipsychiatry views, not only rejecting psychiatry in relation to ME/CFS, but also conducting personal attacks on those professionals who are involved in scientific research and review that come to opposing conclusions that are not aligned with these antipsychiatry views. ${ }^{25}$ A biopsychosocial perspective seems to be the only view that has been consistently rejected by these patient 'activists', with indeed almost every other explanation being theorised as avenues for causation for ME/CFS including the use of pesticides, radiation, diet, allergies, vitamin deficiencies, mitochondrial disorders, infection and so on-most of them mutually incompatible except that none of them are 'psychiatric'. There is a suggestion in the document itself that there were antipsychiatry views among the stakeholders: 'there was a broad range of possible research areas identified, with the strongly expressed view that it should not be undertaken in relation to psychiatry'. When making recommendations regarding the multidisciplinary teams, the report states that consultant clinicians for a specialist ME/ CFS service could be recruited from 'clinical neurology, rehabilitation medicine or infectious diseases specialties', ${ }^{2}$ with no mention of psychological medicine and the expertise that lies within that field. The ScotPHN authors themselves state they 'recognise the importance of population perceptions and the impact of political processes, 2 when planning and delivering healthcare. However, they do not define which among the many populations they are referring to. The ScotPHN is an example of the pitfalls and indeed tribulations of trying to integrate two mutually incompatible perspectives. It does not seem for example that it reflects the views of the participants in the PACE trial, those who took part in the Action for ME survey, what practising clinicians report from the coal face, or the majority of UK neurologists. ${ }^{9}$ In this context, it is also not clear what political actually means. Does it mean that opinion, particularly when held stridently, should over-rule evidence from other sources, where the provenance and methodology is open to scrutiny? Does it mean that viewpoints that others might find frankly offensive should be given equal weight, and by this we mean the unequivocal antipsychiatry views that would not find favour with the members of MIND, for example, let alone the millions of those who suffer from illnesses that are covertly, and sometimes overtly, stigmatised in the more strident voices of the ME activists?

All of that is open to debate. However, the result as demonstrated in the ScotPHN guidelines is less debatable. If the guidelines were to be implemented and/or widely disseminated, Scottish sufferers from ME/CFS would not be able to make an informed choice about treatment, let alone access the same treatments as patients in other parts of the UK.

The accuracy of guidelines and any reviews that are focused on providing information and guiding processes for commissioning and legislation is paramount. These informative documents should be produced regardless of ideological perspectives, and should provide guidance based on what is known, with balance and representation of a range of views, essentially providing choice for patients and clinicians based on sound evidence. While not being prescriptive, guidelines and assessments should be focused on what may benefit patients. Therefore, although guidelines and reports should attempt to integrate moderated 'human views' of the subjective experience into the literature, this should not skew or outweigh the scientific evidence base. There has been an apparent exclusion of a large and helpful wealth of literature, and this cannot be a basis for a review of an impartial evidence base. In this case, the scientific literature-the world of cohort studies, randomised controlled trials and a hierarchy of evidence and critical appraisal-appears to have been downgraded in favour of one particular subjective experience. This is of detriment to not only those patients themselves, but also to the less likely to be heard and probable majority of patients and clinicians, who are relying on guidance and reviews to inform them about what conclusions may be helpful and may be limited in choice by the treatment options recommended. No clinician could enforce an unacceptable treatment on an ME/CFS sufferer. However, making it harder for patients to access treatments that may be of benefit, and similarly for clinicians to be able to offer those treatments, is unhelpful.

\section{IS THIS A UNIQUE SITUATION?}

Some might say that the chosen area that the authors have used to discuss this dilemma in convergence of the scientific and narrative is replete with controversy, with a reputation for intolerance and stridency. That may be true, but it is not unique.

Something very similar, for example, is seen in the debate around 'chronic cerebrospinal venous insufficiency' (CCSVI) and multiple sclerosis (MS). This was sparked off by a paper in which CCSVI was hypothesised as a cause of MS. ${ }^{26}$ There were many faults with the original study that claimed to find an association, the most obvious flaws being that it was not blinded and did not have a comparison group. Despite this, the group went on to devise a 'treatment' intervention which involved surgical balloon angioplasty or stent placement of 'affected' veins ${ }^{27}$; this paper was published within months of the study looking at the hypothesis of causation. Patients with MS searching for further clarification of cause and treatment for MS hailed this a breakthrough, and some surgeons and clinicians carried out these procedures; unfortunately, this resulted in several serious complications and patient deaths related to the intervention. ${ }^{28} \mathrm{~A}$ Lancet review article stated 'the hypothesis is yet to be proven, let alone the safety and efficacy of the intervention... (patients are subjected to) a grave risk without any evidence that the procedure would help treat MS... there is widespread criticism of the media hype associated with the CCSVI hypothesis (which has) caused whirlwind of exaggerated claims and expectations... The premature promotion of the CCSVI hypothesis has led to a situation in which many patients with MS seek venous testing, and, if the result turns out to be abnormal, endovascular interventions'. ${ }^{28}$ In response, NICE recently reviewed the current situation, which states that 'evidence on the efficacy of percutaneous venoplasty for CCSVI for MS is inadequate in quality and quantity. Therefore, this procedure should only be used in the context of research'. ${ }^{29}$ Many patient groups are disregarding this evidence-based medicine and persisting in requesting the procedure based on an unproven theory. ${ }^{30}$

This debate is also true for 'chronic Lyme disease'. In this instance, some patient groups have strongly endorsed the concept of a persistent disease associated with perpetuation of Borrelia bacteria which causes ill-defined but chronic disabling symptoms in the absence of any clinical signs and/or accepted diagnostic laboratory tests. The results are that patients many end up taking long-term antibiotic treatments of little or no benefit but great cost, while prominent patient groups continue to disbelieve and mistrust most of the medical community regarding Lyme disease and frequently seek to discredit evidence-based guidelines, sometimes by resorting to immoral and unethical tactics ${ }^{31}$ in an attempt to distort this evidence base. This had led to considerable pressure on authorities to sanction treatments for which there is no proven efficacy. A 
2011 Lancet commentary concluded that 'activists, through public appeal and political lobbying, have managed to divert attention away from existing evidence-based medicine...there is a serious concern that they will further endanger the public's health unless physicians, scientists, government leaders and the media firmly stand up for an evidence-based approach...that is based on high-quality scientific studies'. ${ }^{32}$

In conclusion, these are examples of a less than helpful interaction between politics and science, and one in which the former has outweighed the latter. The issue is important; it is not an example of patients versus doctors-far from it. It is about the challenge of accurately reflecting and giving due weight to patient views in the discourse, when there is no consensus among those either. The programme of evidence-based medicine is based on the premise that it is possible through reason, logic and debate to take what at first sight seems to be discordant views within the medical literature, and to follow a transparent and reproducible process seeking to understand the causes of the discrepancies, and resolve them via this methodology to arrive at conclusions. However, there seems to be no equivalent process for the other side of the process, to blend this with the views of patients, their narratives, perspectives and insights. Where these all point in the same direction, there is no problem, but where they clearly do not, the examples chosen here show the limitations of the process. An alternative approach in these situations is to highlight areas of consensus, while recognising areas of debate. It is no easy task-to ensure tolerance, allow all shades of opinion to be represented, and then have a transparent and reasonable way of resolving conflict where it continues to exist between evidence that satisfies criteria developed over many years and the views of a particular stakeholder, so that the best interests of all groups are represented. Perhaps that is an impossible task, in which case it is essential that we recognise and acknowledge when the process has failed in order to ensure that the media, health service planners and, most important of all, patients are given a fair representation of the evidence base. After that, they can make up their own minds.

Contributors CS: completed literature search, drafted article, made final revisions to article. SW: revision of article for intellectual content, made final revisions and gave final approval of the article published. SW was the lead author on the first paper proposing a cognitive behavioural model of CFS in 1989, and subsequently led one of the teams that developed and tested CBT for CFS in the following years. He moved away from CFS research around ten years ago, but remains clinically active seeing patients in the CFS service. On the basis of his knowledge of the research and his extensive clinical experience over two decades he does believe that CBT is helpful in the management of CFS.

Funding None.

Competing interests None.

Provenance and peer review Commissioned, not externally peer reviewed.

\section{REFERENCES}

1 Appraisal of Guidelines for Research and Evaluation II Next Steps Consortium. The AGREE II Instrument (Electronic Version). 2009. http://www.agreetrust.org (accessed 6 Jun 2012)

2 Mackie P, Dougall R, Conacher A. Health Care Needs Assessment of Services for people living with ME-CFS, Scottish Public Health Network. 2010. http://www. scotphn.net/pdf/Final report web version 271110.pdf (accessed 11 Mar 2012).

3 Mackie P, Dougall R, Conacher A. Health care needs assessment of services for people living with ME-CFS- short version for patients, Scottish Public Health Network. 2011. http://www.scotphn.net/pdf/ME-CFS_short_report_-_2011_09_09_ Final.pdf (accessed 11 Mar 2012).

4 Carruthers BM, Jain AK, Meirleir KL, et al. Myalgic encephalomyelitis/Chronic Fatigue Syndrome; Clinical Working Case definition, Diagnostic and Treatment Protocols. J Chron Fatigue Syndr 2003;11:1-115.

5 Valeant. Products: Neurology and Other. 2011. http://www.valeant.com/products/ neurology/index.jsp (accessed 27 Feb 2012).
6 National Institute for Health and Clinical Excellence (NICE). Chronic Fatigue Syndromw/myalgic encephalomyelitis (or encephalopathy), NICE clinical guideline 53. 2007. http://www.nice.org.uk/nicemedia/live/11824/36193/36193.pdf (accessed 2 Jan 2012).

7 Scottish Neuroscience Council. Scottish Good Practice Statement on ME-CFS; Comments on the Canadian Consensus Document. Clinical Quick Reference Guide Working Group. 2010. http://www.cathcartmesupportgroup.org.uk/resources/Alan Carson_Clinical_Review_Group.pdf (accessed 28 Feb 2012).

8 The Scottish Government. Scottish Good Practice Statement on ME-CFS. 2010. http://www.show.scot.nhs.uk/App_Shared/docs/ClinicalGuide.pdf (accessed 2 Jan 2012).

9 Sharpe M. Chronic Fatigue Syndrome; Neurological, mental or both. J Psychosom Res 2011;70:498-9.

10 Perderson BK, Saltin B. Evidence for prescribing exercise as therapy in chronic disease. Scand J Med Sci Sports 2006;16(Suppl. 1):3-63.

11 Whiting $\mathrm{P}$, Bagnall AM, Sowden AJ, et al. Interventions for the treatment and management of chronic fatigue syndrome: a systematic review. J Am Med Assoc 2001;286:1360-8.

12 Edmonds M, McGuire H, Price J. Exercise therapy for Chronic Fatigue Syndrome (Cochrane Review). The Cochrane Library. Issue 3, Chichester, UK: John Wiley \& Sons, Ltd, 2004.

13 Chambers D, Bagnall AM, Hempel S, et al. Interventions for the treatment, management and rehabilitation of patients with chronic fatigue syndrome/myalgic encephalomyelitis; an updated systematic review. J R Soc Med 2006;99:506-20.

14 NHS Centre for Reviews and Dissemination. Systematic review on the effectiveness of interventions used in the treatment and management of chronic fatigue syndrome/myalgic encephalitis (ME) among adults and children, The NHS Centre for Reviews and Dissemination Report 22. 2002. University of York, http://www.york.ac. uk/inst/crd/cfs.htm (accessed 28 Feb 2012).

15 NHS Centre for Reviews and Dissemination. Effective treatments for CFS/ME, The NHS Centre for Reviews and Dissemination Report 35. 2006. University of York, http://www.york.ac.uk/inst/crd/CRD_Reports/crdreport35_summ.pdf (accessed 28 Feb 2012).

16 Prins JB, van der Meer JWM, Bleijenberg G. Review; Chronic Fatigue Syndrome. The Lancet 2006;367:346-55.

17 Castell BD, Kazantzis N, Moss-Morris RE. Cognitive behavioural therapy and graded exercise for Chronic Fatigue Syndrome: a meta-analysis. Clin Psychol Sci Pract 2011;18:311-24

18 White PD, Goldsmith KA, Johnson AL, et al. Comparison of adaptive pacing therapy, cognitive behaviour therapy, graded exercise therapy, and specialist medical care for chronic fatigue syndrome (PACE), a randomised trial. The Lancet 2011;377:823-36.

19 R (Fraser) v National Institute for Health and Clinical Excellence (2009) EWHC 452 (Admin); (2009) 107 BMLR 178 (Simon J), England and Wales High Court (Administrative Court) Decisions. http://www.bailii.org/ew/cases/EWHC/Admin/2009/ 452.html (accessed 13 Jan 2012).

20 Twisk FNM, Maes M. A review on Cognitive Behavioural therapy (CBT) and graded exercise therapy (GET) in myalgic encephalomyelitis (ME)/ Chronic Fatigue Syndrome (CFS); CBT/GET is not only ineffective and not evidence-based, but also potentially harmful for many patients with ME/CFS. Neuroendocrinol Lett 2009;30:284-99.

21 Bleijenberg G, Knoop H. Chronic Fatigue Syndrome; where to PACE from here? The Lancet 2011;6736:60172-4.

22 Mayo Clinic. Chronic Fatigue Syndrome- Treatments and drugs. 2011. http://www. mayoclinic.com/health/chronic-fatigue-syndrome/DS00395/ DSECTION=treatments-and-drugs. (accessed 22 Dec 2011).

23 Bupa. Treatment of Chronic Fatigue Syndrome. 2010. http://www.bupa.co.uk/ individuals/health-information/directory/c/ hi-chronic-fatigue-syndrome\#textBlock201829 (accessed 22 Dec 2011).

24 Interaction. You say "Save our services!". Interaction 2011;77:4-5.

25 Hawkes N. Dangers of research into Chronic Fatigue Syndrome. $\mathrm{Br}$ Med J 2011;342:d3780.

26 Zamboni P, Galeotti R, Menegatti E, et al. Chronic cerebrospinal venous insufficiency in patients with multiple sclerosis. J Neurol Neurosurg Psychiatry 2009;80:392-9.

27 Zamboni P, Galeotti R, Menegatti E, et al. A prospective open-label; study of endovascular treatment of chronic cerebrospinal venous insufficiency. I Vasc Surg 2009:50:1348-58.e3.

28 Qiu J. Venous abnormalities and multiple sclerosis: another breakthrough claim? Lancet Neurol 2010;9:464-5.

29 National Institute for Health and Clinical Excellence. Percutaneous venoplasty for chronic cerebrospinal venous insufficiency for multiple sclerosis, NICE interventional procedure guidance 420. 2012.http://www.nice.org.uk/nicemedia/live/13064/58610/ 58610.pdf (accessed1 Apr 2012)

30 MS-CCSVI-UK. DIY for CCSVI. 2012. http://www.ms-ccsvi-uk.org/home/diy-for-ccsvi html (accessed 1 Apr 2012).

31 Grann D. Stalking Dr. Steere over Lyme disease. US Edition, USA: New York Times, 2001. http://www.nytimes.com/2001/06/17/magazine/17LYMEDISEASE.html (accessed 1 Apr 2012).

32 Auwaertar PG, Bakken JS, Dattwyler RJ, et al. Antiscience and ethical concerns associated with advocacy of Lyme disease. Lancet Infect Dis 2011;11:713-19. 